\title{
On certain cosmological relics of the early string dynamics
}

\author{
Nikolaos A. Batakis* \\ Department of Physics, University of Ioannina \\ GR-45110 Ioannina, Greece
}

\begin{abstract}
The tracing of cosmological relics from the early string dynamics may enhance the theory and provide new perspectives on the major cosmological problems. This point is illustrated in a leading-order Bianchi-type $V I I_{0}$ string background, wherein spatial isotropy can be claimed as such a relic, albeit a gross one. A much finer relic, descending from a premordial gravitational wave, could be retrieved from its imprint on the small-scale structure of the cosmic microwave background. In spite of the absence of conventional inflation, there is no horizon problem thanks to the presence of an equally fundamental mixmaster dynamics. Implications and certain new perspectives which thus arise for the more general problem of cosmological mixing are briefly discussed.
\end{abstract}

*e-mail: nbatakis@cc.uoi.gr 


\section{Introduction}

Within the anticipated span of string theory, all observables should be viewable as relics of the early dynamics, albeit not always easily tracable ones. In the context of string cosmology in particular, one would be justified to expect observables which, in addition to measurable differences from the conventional (general relativistic) description, would also provide new perspectives on the major cosmological problems. We do not yet have a physically acceptable space-time realization of whatever is anticipated as a uniquely acceptable conformal field theory or at least a reasonable 'strong-field' (namely excact to all orders in $\alpha^{\prime}$ ) solution, which will illuminate the short-distance structure of space-time [1]-[3]. Nevertheless, whatever this primordial picture may be, we know that it will quickly (say, within the first $10^{-40} \mathrm{sec}$ ) start evolving towards the classical-gravity regime of a 4D space-time, as exemplified by the string effective action [4, [5]. Taking also into account the existing cosmological phenomenology, one could possibly identify string relics which would be virtually independent of the precise short-distance formulation of the theory. Such relics would most profitably be searched for in the cosmic microwave background profiles and charts, which provide par excellence the most accurate cosmological data [6]. A fundamental cosmological problem likely to be affected by such considerations is the composite one of mixing, whose various facets are better known individually as the horizon problem, the isotropy and entropy problems, the sensitivity to initial conditions (related to the flatness problem) and so on [7]-11].

The above remarks also summarize the motivation and general objective of this paper. A Bianchi-type $V I I_{0}$ spacetime realized as a leading-order in $\alpha^{\prime} 4 \mathrm{D}$ string background illustrates the above issues, as it turns out to be modestly realistic and rich in that respect. The spatial isotropy, eventually established by a gravity-wave driven mixmaster mechanism, can be claimed as a gross relic of the early string dynamics. A descendent of that wave also survives as a much finer relic, with valuable data for string theory and the cosmological mixing problem. Our main results will be presented in sections 2,3 and further discussed in 4 .

To establish notation, we recall that the low-energy string effective action in $4 \mathrm{D}$ in the Einstein frame with vanishing cosmological constant may be expressed as

$$
S_{e f f}=\int d^{4} x \sqrt{-g}\left(R+2 g^{\mu \nu} \frac{\partial_{\mu} S_{+} \partial_{\nu} S_{-}}{\left(S_{+}-S_{-}\right)^{2}}\right),
$$

with the non-linear $S L(2, R) / U(1) \sigma$-model variables expressed as $S_{ \pm}=b \pm i e^{\phi}$ in terms of the axion/dilaton pair. The one-loop beta-function equations for conformal invariance follow from 
(11) as

$$
\begin{aligned}
R_{\mu \nu}+\frac{2}{\left(S_{+}-S_{-}\right)^{2}} \partial_{\mu} S_{+} \partial_{\nu} S_{-} & =0 \\
\partial_{\mu}\left(g^{\mu \nu} \frac{\sqrt{-g} \partial_{\nu} S_{\mp}}{\left(S_{+}-S_{-}\right)^{2}}\right) \pm 2 \sqrt{-g} g^{\mu \nu} \frac{\partial_{\mu} S_{+} \partial_{\nu} S_{-}}{\left(S_{+}-S_{-}\right)^{3}} & =0 .
\end{aligned}
$$

\section{The Bianchi-type $V I I_{0}$ string background}

Any 4D space-time metric can always be organized as diagonal in a properly chosen (generally non-holonomic) basis. The particular basis of 1 -forms $\left\{\sigma^{i}, i=1,2,3\right\}$ employed here is invariant under the left action of the Bianchi-type $V I I_{0} 3$-parameter group of transitive Killing isometries [7], [5], namely with structure constants suplied by the defining relations

$$
d \sigma^{1}=-\sigma^{2} \wedge \sigma^{3}, \quad d \sigma^{2}=-\sigma^{3} \wedge \sigma^{1}, \quad d \sigma^{3}=0 .
$$

The group generators are best realized as three independent killing vectors which define three principal directions of anisotropy and span each one of a continuous family of homogeneous 3D spacelike hypersurfaces $\Sigma^{3}$ parametrized by $t$. More explicitly, our metric will have the form [5]

$$
d s^{2}=-d t^{2}+a_{1}(t)^{2}\left(\sigma^{1}\right)^{2}+a_{2}(t)^{2}\left(\sigma^{2}\right)^{2}+a_{3}(t)^{2}\left(\sigma^{3}\right)^{2},
$$

wherein the metric coefficients $a_{i}$, just like the $b, \phi$ fields, are functions of the proper (co-moving) time $t$ only. One set of explicit holonomic-coordinate $x^{i}$ realizations for the $\sigma^{i}$ resulting from the defining relations (幽 (with $k$ an arbitrary real constant) is

$$
\sigma^{1}=\cos \frac{k}{2} x^{3} d x^{1}+\sin \frac{k}{2} x^{3} d x^{2}, \quad \sigma^{2}=-\sin \frac{k}{2} x^{3} d x^{1}+\cos \frac{k}{2} x^{3} d x^{2}, \quad \sigma^{3}=d x^{3} .
$$

These expressions are subject to any transformation which preserves the structure of (4). With the new coordinate time $\tau$ defined in terms of the mean radius $a$ as

$$
d t=a_{1} a_{2} a_{3} d \tau=a^{3} d \tau
$$

so that $a^{-1} d a / d t$ is the mean Hubble constant of any comoving volume element in $\Sigma^{3}$, and with a prime standing for $d / d \tau$ we may re-express (2) as

$$
\left(\ln a_{1}^{2}\right)^{\prime \prime}+a_{1}^{4}-a_{2}^{4}=0, \quad\left(\ln a_{2}^{2}\right)^{\prime \prime}+a_{2}^{4}-a_{1}^{4}=0, \quad\left(\ln a_{3}^{2}\right)^{\prime \prime}-\left(a_{1}^{2}-a_{2}^{2}\right)^{2}=0 .
$$

The $a_{i}(\tau)$ are also subject to the initial-value equation

$$
\left(\ln a_{1}^{2}\right)^{\prime}\left(\ln a_{2}^{2}\right)^{\prime}+\left(\ln a_{2}^{2}\right)^{\prime}\left(\ln a_{3}^{2}\right)^{\prime}+\left(\ln a_{3}^{2}\right)^{\prime}\left(\ln a_{1}^{2}\right)^{\prime}-\left(a_{1}^{2}-a_{2}^{2}\right)^{2}=A^{2},
$$


essentialy the 00 equation in the set (2). The pair of Eqs. (3) can be written as

$$
b^{\prime}+A e^{2 \phi}=0, \quad \phi^{\prime \prime}+A^{2} e^{2 \phi}=0
$$

with general solution

$$
b=\left(\frac{N}{A}\right) \frac{\sinh (N \tau)+\sqrt{1-\frac{A^{2}}{N^{2}}} \cosh (N \tau)}{\cosh (N \tau)+\sqrt{1-\frac{A^{2}}{N^{2}}} \sinh (N \tau)}, e^{-\phi}=\cosh (N \tau)+\sqrt{1-\frac{A^{2}}{N^{2}}} \sinh (N \tau),
$$

where $A, N$ are constants and where two more constants of integration have been absorbed to fix the origins of $b, \phi$. The totally antisymmetric tensor wherefrom $b$ descends may be equivalently expressed as the 3 -form $H=-e^{-\phi}(d b)^{*}$, namely as

$$
H=A \sigma^{1} \wedge \sigma^{2} \wedge \sigma^{3}=A d x^{1} d x^{2} d x^{3},
$$

which also identifies $A$ as the magnitude of $H$ per unit of invariant volume in $\Sigma^{3}$. We also note that the $\sigma$-model contributes in (1) as a source to the gravitational field with the energymomentum tensor

$$
\kappa^{2} T_{\mu \nu}=-\frac{S_{+}^{\prime} S_{-}^{\prime}}{\left(S_{+}-S_{-}\right)^{2}} \operatorname{diag}\left(1, g_{i j}\right) .
$$

The general solution to Einstein's equations (8) may be expressed as

$$
a_{1}^{2}=e^{2 P \tau+f}, \quad a_{2}^{2}=e^{2 P \tau-f}, \quad a_{3}^{2}=e^{2 P \tau+h}
$$

still subject to (9), with $P$ a positive constant and $f, h$ solutions to the coupled system

$$
\begin{aligned}
f^{\prime \prime}+2 e^{4 P \tau} \sinh 2 f & =0, \\
h^{\prime \prime}-4 e^{4 P \tau}(\sinh f)^{2} & =0 .
\end{aligned}
$$

Special solutions thereoff with $S O(2)$ and $S O(3)$ isotropy can be easily obtained. The latter case is realized by the trivial solution $f=0, h=0$ of (15,16) as

$$
a_{1}=a_{2}=a_{3}=a=e^{P \tau} \sim t^{1 / 3} \quad P^{2}=\frac{1}{12} A^{2},
$$

which is just a flat FRW background with axion/dilaton configurations given by (11). This spatially isotropic configuration will be of special interest to our present considerations, not so much as a solution by itself but rather as the asymptotic limit of any other solution, as we will see. Although as far as we are aware the above results are generally new, part of them reproduces some basic aspects of the Bianchi-type $V I I_{0}$ geometry already known in a general relativistic context [10, [11], as well as more recent results in string cosmology [4, [5]. 


\section{Mixing and relics}

The mixing process and relics mentioned are aspects of the dynamics described by the solution (14) subject to (9) together with the non-linear system (15, 16). This exhibits an ergodic behavior off the initial singularity, which has been studied as a rather typical aspect of Einstein's equations in general, as well as in the particular case of the Bianchi-type $V I I_{0}$ geometry and its stability [10]-[12]. The special interest of (17), associated with the trivial solution $f=0$ of (15) as mentioned, lies in the fact that it is an attractor solution, which means that it is the only analytic one (at least in an extented domain) and that any other solution of (15) will eventually approach $f=0$ at $\tau \rightarrow+\infty$. The actual descend of any $f$ towards the $f=0$ solution is realized following a series of initial adjustments to generally lower values, whereafter $f$ enters an oscillatory evolution (around the $f=0$ limit) with monotonically decreasing amplitudes and increasing frequencies. This behavior can be shown and stated more rigorously if we restrict ourselves to sufficiently large times, namely values of $t$ or $\tau$ such that the factor sinh $2 f$ in (15) is practically equal to $2 f$. Then, under a re-definition of the independent variable as $x=e^{2 P \tau} / P$, (15) is transformed to

$$
x \frac{d^{2} f}{d x^{2}}+\frac{d f}{d x}+x f=0 .
$$

This is the zeroth-order Bessel equation, whose general solution is a linear combination of the zeroth-order Bessel functions $J_{0}(x)$ and $Y_{0}(x)$. From their known behavior we immediately see that their approach to zero confirms the generally claimed pattern while, for sufficiently large $x$ in particular, we find

$$
f(\tau)=F e^{-P \tau} \sin \left(\frac{e^{2 P \tau}}{P}\right),
$$

where $F$ is a constant. The behavior of $h$ can be recovred from (16), still subject to (9) which we may re-express as

$$
f^{\prime 2}-4 P h^{\prime}+4 e^{4 P \tau}(\sinh f)^{2}=12 P^{2}-A^{2} .
$$

We note, however, that we may set each side of (20) to zero if $A \neq 0$ (cf. also (17)).

The zeroes (namely the sign-changes) of $f$ give rise to a succession of Kasner-like bounces, in rather striking resemblance to the mixmaster prototype [8], as can be seen in relation to (14,200). Although this will be discussed later on, we may already argue that the hence emerging isotropy can be claimed as a relic of the early string dynamics by observing that (i) The possibility of isotropy is in the first place due to that dynamics, because in a conventional general relativistic vacuum, or if we just turn-off the axion scalar by setting $A=0$ in initial-value equation (9), 
we immediately see that isotropy is forbidden. (ii) Once possible, isotropization will eventually prevail as a result of its association with the $f=0$ attractor solution, seen on physical grounds as an evolution of the model towards lower-energy-of-anisotropy configurations.

We now observe that the metric (5) written in terms of the holonomic coordinates (6) together with (14) is

$$
d s^{2}=-d t^{2}+g_{i j} d x^{i} d x^{j}
$$

wherein the part of the metric describing the geometry and evolution of the homogeneous $\Sigma^{3}$ sections (with $x^{3}=z$ for brevity) is

$$
g_{i j}=e^{2 P \tau}\left(\begin{array}{ccc}
e^{f} \cos ^{2} \frac{k}{2} z+e^{-f} \sin ^{2} \frac{k}{2} z & \left(e^{f}-e^{-f}\right) \cos \frac{k}{2} z \sin \frac{k}{2} z & 0 \\
\left(e^{f}-e^{-f}\right) \cos \frac{k}{2} z \sin \frac{k}{2} z & e^{f} \sin ^{2} \frac{k}{2} z+e^{-f} \cos ^{2} \frac{k}{2} z & 0 \\
0 & 0 & e^{h}
\end{array}\right) .
$$

This type of metric has been already examined in a general-relativistic context and in particular within a Bianchi-type $V I I_{0}$ setting, as describing a flat background modulated by a standing gravitational wave or, equivalently, as a pair of gravitational waves propagating in $\Sigma^{3}$ in opposite $( \pm z)$ directions [10]. A similar decomposition of (22) will be here directly related to the two relics mentioned in the introduction. Indeed, in a decomposition of the form

$$
g_{i j}=g_{i j}^{(0)}+g_{i j}^{(1)}
$$

we may easily extract from (22) the flat-bacground metric

$$
d s_{0}^{2}=-d t^{2}+g_{i j}^{(0)} d x^{i} d x^{j}
$$

with

$$
g_{i j}^{(0)}=e^{2 P \tau}\left(\begin{array}{ccc}
\cosh f & 0 & 0 \\
0 & \cosh f & 0 \\
0 & 0 & e^{h}
\end{array}\right),
$$

on top of which we have as a 'perturbation' (which, of course, is neither approximate nor necessarily small) the remaining part in (22), namely

$$
g_{i j}^{(1)}=e^{2 P \tau} \sinh f\left(\begin{array}{ccc}
\cos k z & \sin k z & 0 \\
\sin k z & -\cos k z & 0 \\
0 & 0 & 0
\end{array}\right) .
$$

The latter configuration is (i) traceless, (ii) covariantly constant and (iii) an eigenfunction of the wave operator, with all operations taken with repect to the flat background (24). While (i),(ii) can be easily verified, to show (iii) we observe that

$$
{ }^{(3)} \nabla^{2} g_{i j}^{(1)}=-\left(k e^{-P \tau-h / 2}\right)^{2} g_{i j}^{(1)},
$$


so that the eigenvalue in (27) is minus the wavenumber $(2 \pi / \lambda)$ squared. The amplitudes of the wave translated within $\Sigma^{3}$ along $z$ are simultaneously differentially-rotated in the $x^{1}, x^{2}$ plane by the local $S O(2)$ element contained in (26). For more direct calculations we may exploit the invariance of (26) under increments of $z$ by $\pm \pi$, expressing the resulting spacelike displacement by means of (21), to find

$$
\lambda=\frac{2 \pi}{k} e^{P \tau+h / 2}=\frac{\pi P}{k} e^{-2 P \tau} l_{z}
$$

This result confirms our earlier identification of $-(2 \pi / \lambda)^{2}$ as the eigenvalue in the rhs of (27). The second equality in (28) relates the wavelength $\lambda$ to $l_{z}$, namely to the scale of the horizon in the $z$ direction (along which the wave is developed), defined as

$$
l_{z}=a_{3} \int d t / a_{3}=\frac{1}{2 P} e^{3 P \tau+h / 2}=\frac{1}{2 P} a^{3} .
$$

These exact results also apply at the $\tau \rightarrow+\infty$ limit where the horizon scale $l_{z}$ becomes of the order of the cosmic time $t$ as defined by (7). More explicitly, we find

$$
l_{z} \rightarrow \frac{1}{2 P} e^{3 P \tau} \sim t, \quad \lambda \rightarrow \frac{2 \pi}{k} e^{P \tau} \approx \frac{2 \pi}{k} a, \quad g_{i j}^{(0)} \rightarrow e^{2 P \tau} \operatorname{diag}(1,1,1) .
$$

The amplitude in (26) does not vanish (in spite of the presence of the $f$ factor which vanishes at that limit) so the wave survives as the second (and much finer) relic mentioned earlier. Utilizing the asymptotic behavior of $f$ in (19) we find

$$
\begin{aligned}
g_{i j}^{(1)} \rightarrow \quad F e^{P \tau} \sin \left(e^{2 P \tau} / P\right)\left(\begin{array}{ccc}
\cos k z & \sin k z & 0 \\
\sin k z & -\cos k z & 0 \\
0 & 0 & 0
\end{array}\right)= \\
F t^{1 / 3} \sin \frac{2 \pi}{\lambda} t\left(\begin{array}{ccc}
\cos k z & \sin k z & 0 \\
\sin k z & -\cos k z & 0 \\
0 & 0 & 0
\end{array}\right),
\end{aligned}
$$

which will be further discussed in the next section.

\section{Discussion and conclusions}

In addition to the ergodicity of the above solution at sufficiently early times, we also expect higher-order in $\alpha^{\prime}$ corrections as $\tau \rightarrow-\infty$. In other words, there is an appropriately defined time $\tau_{0}$ near the initial singularity, below which the present model will have to be trancated and matched to that higher-order solution. Due to its ergodicity in that regime, the present solution will presumably undergo an almost dense sample of values there, virtually including the ones 
to be matched at $\tau_{0}$ (for an at least $C^{1}$ junction). From that time onwards, the evolution can be seen to span three epochs, delimited by the instant $\tau_{\text {iso }}$ (to be formally defined in the sequel) at which isotropization has practically commenced.

The initial epoch involves times $\tau$ with $\tau_{0} \leq \tau \ll \tau_{\text {iso }}$ and it is characterized by virtually arbitrary initial conditions, intractable (ergodic) dynamics which involves solutions of (15) with $|f| \gg 1$, and arbitrarily large anisotropy. The wavelength $\lambda$ is also very large compared to $l_{z}$, namely no full wavelength $\lambda$ has yet been formed within the young horizon in $\Sigma^{3}$ (quantified by $l_{z}$ and $a$, respectively, and subject to (29)) as can be seen from (28). According to (14), the sign reversals of $f$ (later to be counted by the number of zeroes of the Bessel functions from (18)) cause a large number of Kasner-like bounces along the principal directions of anisotropy, as mentioned earlier. The bounces induce adequate mixing and isotropization while the model is been driven towards configurations with lower energy of anisotropy, together with the generation of a considerable amount of entropy, as one can deduce from (14 16) and (28) 31). This mechanism seems to offer a viable resolution not only to the horizon problem but to the mixing problem in general, essentially within the objectives of the Mixmaster prototype [8]-[11]. Such a development could be a wellcome alternative, particularly in view of the apparently serious difficulties faced by the more conventional inflationary schemes [13].

After the initial but well before the model reaches its final epoch realized at $\tau \gg \tau_{\text {iso }}$, there is an intermediate epoch chracterized by times $\tau \approx \tau_{\text {iso. }}$. The wavelength $\lambda$ continues to grow with the overall Hubble expansion while, simultaneously, it is shrinking with respect to the (faster expanding) horizon $l_{z}$, as seen from (28 30). Thus, the initial-epoch $\lambda \gg l_{z}$ relationship (on its way to inevitably change to $\lambda \ll l_{z}$ in in the final epoch) will pass through the equality $\lambda=l_{z}$, at an instant of time which we may now formally define as $t_{\text {iso }}$, where we also have $|f| \approx 1$. The emerging picture in this intermediate epoch is that all points within the horizon have begun to correlate as elements of a common dynamics towards the formation of the above single gravitational wave in $\Sigma^{3}$. We may thus claim that this equivalence and the associated isotropization will have been attained to first order (in an approximation scheme having nothing to do with the $\alpha^{\prime}$ expansion) as soon as the first full wavelength is created within the horizon, namely when $\lambda=l_{z}$ at $t=t_{\text {iso }}$. The second-order approximation is realized with the formation of the second full wavelength within the horizon, and so on. The time required for the realization of each successive order is obviously decreasing, being inversely proportional to the monotonically increasing frequency of oscillations of $f$.

During the final epoch $\left(t \gg t_{\text {iso }}\right)$, the background metric (25) evolves smoothly towards its 
flat isotropic limit (17) and has by then completely overgrown the wave (26), now described by (31). The axion/dilaton pair, which generally develops according to (11), has driven the model to lower couplings [14] with $e^{\phi}$ diminishing as $e^{-|N| \tau}$ when $\tau \rightarrow \infty$. The usual cosmological parameters are given by (or may be derived from) the results (19,30,31) and the space-time dynamics can be safely treated here in the adiabatic approximation. The amplitude, wavelength and frequency of the wave (31) are easily found to be constant multiplied by $e^{P \tau} \sim t^{1 / 3}$ factors, carrying the overall cosmological (Hubble) expansion common to all scales and couplings. The actual value of the amplitude $F$ in particular, expected to be quite small, is of importance because it determines the relative magnitude of (31) (considered as a perturbation) with respect to the background metric. Rather than being an arbitrary overall factor (if erroneously associated with the homogeneous equation (18)), $F$ actually descends from the non-linear (15) and it is therefore expressible in terms of $A=2 \sqrt{3} P$ and numerical constants. $F$ will also specify the scale of the energy density stored in the relic wave, and its relation to the energy of anisotropy in (13) wherefrom it has been effectively drawn.

Contrasted to any anticipated stochastic (gravitational 'black body' radiation) background [15], the single gravitational wave which survives in the present model should have a distinct impact and signature in relatively recent times, in paricular at the electromagnetic radiation decoupling and thus on the geometry of the hypersurface of recombination [3, [9]. It follows that the parameters of such a wave could in principle be uncovered from multipole moments or fine modulations over the nearly-perfect isotropic microwave background [6], [16]. It is hoped that, in spite of its shortcommings, the present model is sufficiently realistic to be exploitable in that respect. If successfully carried out, such a programme could provide quantitative estimates on the axion/dilaton scalars and other elements of the early string dynamics. 


\section{References}

[1] E. Fradkin and A. Tseytlin, Nucl. Phys. B261 (1985)1;

C. Callan, D. Friedan, E. Martinec and M. Perry, Nucl. Phys. B262 (1985)593.

[2] A. A. Tseytlin, 'Exact solutions of closed string theory', preprint Imperial/TP/94-95/28, hep-th/9505052.

[3] E.W. Kolb and M.S. Turner, The Early Universe: Reprints, Addison-Wisley, N.Y., 1988; The Early Universe, Addison-Wisley, N.Y., 1990.

[4] A. Tseytlin and C. Vaffa, Nucl. Phys. B327 (1992)443, and additional references cited therein.

[5] N.A. Batakis and A.A. Kehagias, Nuc.Phys. B 449 (1995) 248;

N.A. Batakis, Phys. Lett. B 353 (1995) 39.

[6] G. F. Smoot et al., ApJ. Lett. 396 (1992) L1;

A. de Oliveira-Costa and G. F. Smoot, ApJ. 448 (1995) 447 and additional references cited therein.

[7] G.F.R. Ellis and M.A.H. MacCallum, Commun. Math Phys. 12 (1969)108; 19 (1970)31;

M.A.H. MacCallum, 'Anisotropic and Inhomogeneous Relativistic Cosmologies', in General Relativity- An Einstein Centenary Survey, eds. S. W. Hawking and W. Israel, Cambridge Univ. Press, Campridge, 1979;

M. Ryan and L. Shepley, Homogeneous Relativistic Cosmologies, Princeton Univ. Press, Princeton, 1975.

[8] C.W. Misner, Phys. Rev. Lett. 22 (1969)1071.

[9] C.W. Misner, K.S. Thorn, J.A. Wheeler, Gravitation Freeman, N.Y., 1973;

S. Weinberg, Gravitation and cosmology, Wiley, N.Y., 1972.

[10] M. Demianski and L.P. Grishchuk, Commun. Math. Phys. 25 (1972) 2333;

V.N. Lukash, Sov. Phys. JETP 40 (1975) 792.

[11] J. D. Barrow, Phys. Rep. 85 (1982)1;

J. D. Barrow and D. H. Sonoda, Phys. Rep. 139 (1986)1. 
[12] P. Holmes, Phys. Rep. 193 (1990)137.

[13] R. Brustein and P.J. Steinhardt, Phys. Lett. B 302 (1993) 196.

[14] E. Kiritsis and C. Kounnas, 'Infrared-regulated string theory and loop corrections to coupling constants' preprint CERN-TH/95-172, LPTENS-95/29, hep-th/9507051.

[15] R. Brustein, M. Gasperini, M. Giovannini and G. Veneziano, 'Relic gravitational waves from string cosmology' preprint CERN-TH/95-144, BGU-PH-95/06, hep-th/9507051.

[16] E. W. Mielke and F. E. Schunk, Phys. Rev. D 52 (1995) 672. 